\title{
Growth mechanisms of GaN microrods for 3D core-shell LEDs: the influence of silane flow
}

\author{
Jana Hartmann ${ }^{*}, 4,5$, Xue Wang ${ }^{3,5}$, Henning Schuhmann ${ }^{1}$, Wanja Dziony ${ }^{2}$, Lorenzo Caccamo ${ }^{5}$, Johannes \\ Ledig $^{4,5}$, Matin Sadat Mohajerani ${ }^{5}$,Tilman Schimpke, ${ }^{3,5}$, Markus Bähr ${ }^{5}$, Gerhard Lilienkamp ${ }^{2}$, Winfried \\ Daum $^{2}$, Michael Seibt' ${ }^{1}$, Martin Straßburg ${ }^{3}$, Hergo-Heinrich Wehmann ${ }^{4,5}$, Andreas Waag ${ }^{4,5}$ \\ ${ }^{1}$ IV. Physikalisches Institut Halbleiterphysik, Georg-August-Universität Göttingen, Friedrich-Hund-Platz 1, 37077 Göttingen, Germa- \\ ny \\ ${ }^{2}$ Institut für Energieforschung und Physikalische Technologien, Technische Universität Clausthal, Leibnizstr. 4, 38678 Clausthal, \\ Germany \\ ${ }^{3}$ Osram Opto Semiconductors GmbH, Leibnizstraße 4, 93055 Regensburg, Germany \\ ${ }^{4}$ Epitaxy Competence Center ec2, Hans-Sommer-Straße 66, 38106 Braunschweig, Germany \\ ${ }^{5}$ Institut für Halbleitertechnik and Laboratory for Emerging Nanometrology, Technische Universität Braunschweig, 38092 Braun- \\ schweig, Germany
}

Received ZZZ, revised ZZZ, accepted ZZZ

Published online ZZZ (Dates will be provided by the publisher.)

Keywords GaN microrods, silane, Si-rich passivation layer, growth model

* Corresponding author: e-mail Jana.Hartmann@tu-bs.de, Phone: +00 495313913 786, Fax: +00 495313915844

The three dimensional growth of GaN structures as a basis for the fabrication of 3D GaN core-shell LEDs has attracted substantial attention in the past years. GaN nanorods or microrods with high aspect ratios can be grown by selective area epitaxy on a $\mathrm{GaN}$ buffer through a $\mathrm{SiO}_{x}$ mask. It has been found earlier that silane substantially initiates vertical growth, with the exact underlying mechanisms being still unclear. Here, the influence of silane on the $3 \mathrm{D} \mathrm{GaN}$ column growth was investigated by performing detailed growth experiments in combination with a thorough surface analysis in order to get insight into these mechanisms. The vertical growth rate is signif- icantly enhanced by high silane fluxes, whereas the saturation of growth rate with the time is reduced. Thus, homogenous GaN columns with an aspect ratio of more than 35 could be achieved. A thin Si-rich layer on the non-polar m-plane facets of the columns has been detected using a combination of transmission electron microscopy, energy dispersive X-ray spectroscopy and Auger electron spectroscopy. This layer is suggested to be the reason for the increase in growth rate, modifying the effective collection range of the species along the sidewalls, and preventing the lateral growth.
1 Introduction The research field of solid state lighting was impelled by light emitting diodes (LEDs) based on $\mathrm{GaN}$, especially because of their superior luminous efficacy and their long lifetime. In order to further increase the luminous flux per wafer area of GaN LEDs, three dimensional (3D) approaches were promoted during the last years. Especially columns of $\mathrm{GaN}$ promise to be a suitable basis for the next generation of LEDs [1],[2] and even for other (opto-)electronic devices like solar cells [3], photo anodes for water splitting [4] or field effect transistors [5],[6]. The enhanced surface area provides a much larger light emitting area and the reduced defect density can lead to higher quality material. Besides, the non-polar sidewalls prohibit the Quantum Confined Stark Effect [7] and therefore thicker quantum wells (QWs) can be grown [8]. External quantum efficiencies of $\mathrm{m}$-plane planar LEDs revealed less droop compared to c-plane LEDs [9]. Thus, the core-shell approach with non-polar m-plane QWs is regarded as a promising way for realization of the next generation of LEDs [10]. In this approach, an n-doped GaN core is surrounded by an InGaN/GaN multi quantum well (MQW) and by a p-doped GaN layer. For device applications it is very important to have a good control over the three-dimensional core growth and to achieve a large as- 
pect ratio, i.e. the height to diameter ratio. In this study, the effects of silane flow on the growth rate in metal organic vapor phase epitaxy (MOVPE) are presented. A moderate silane flow during the growth promotes the vertical growth rate, however, after a certain growth time no or much less additional vertical growth can be observed. For a higher silane flow no such saturation was observed up to $28 \mu \mathrm{m}$ height of the columns. The reasons for this behavior are presently unclear. In this report, a combination of growth experiments and surface analysis with high spatial resolution leads to important insights into the role of silane during the $3 \mathrm{D}$ growth of $\mathrm{GaN}$.

2 Selective area epitaxy The templates for the selective area growth (SAG) of 3D structures are prepared by a $\mathrm{SiO}_{\mathrm{x}}$ mask on a $\mathrm{GaN}$ layer on 2" c-plane sapphire substrates with a $0.2^{\circ}$ off-cut towards the m-plane. The $\mathrm{SiO}_{x}$ layer of about $30 \mathrm{~nm}$ thickness is patterned by photolithography with a hexagonal pattern of holes with a diameter of $800 \mathrm{~nm}$ and a pitch of $2.4 \mu \mathrm{m}$. The MOVPE growth in a vertical showerhead reactor starts with the filling of the opened holes (layer growth conditions) and is continued by the vertical growth of GaN with a flow of $134 \mu \mathrm{mol} / \mathrm{min}$ Trimethylgallium (TMGa), $4.4 \mathrm{mmol} / \mathrm{min}$ ammonia $\left(\mathrm{NH}_{3}\right)$ and varying silane $\left(\mathrm{SiH}_{4}\right)$ flows. To determine the growth rates of the Ga-polar GaN columns, the sample geometry has been accessed by scanning electron microscopy (SEM) The growth times were varied from $300 \mathrm{~s}$ to $1800 \mathrm{~s}$ (Fig. 1 a-d) for growth without silane, from $600 \mathrm{~s}$ to $3600 \mathrm{~s}$ for growth with a constant silane flow of $110 \mathrm{nmol} / \mathrm{min}$ (Fig. 1 e-h) and from $300 \mathrm{~s}$ to $2700 \mathrm{~s}$ for growth with a constant silane flow of $330 \mathrm{nmol} / \mathrm{min}$ (not shown here). The heights of the columns derived from the SEM images are plotted over the growth time in Fig. 2. For the columns grown without and with $110 \mathrm{nmol} / \mathrm{min}$ silane, two linear fits were performed for different time ranges, respectively.
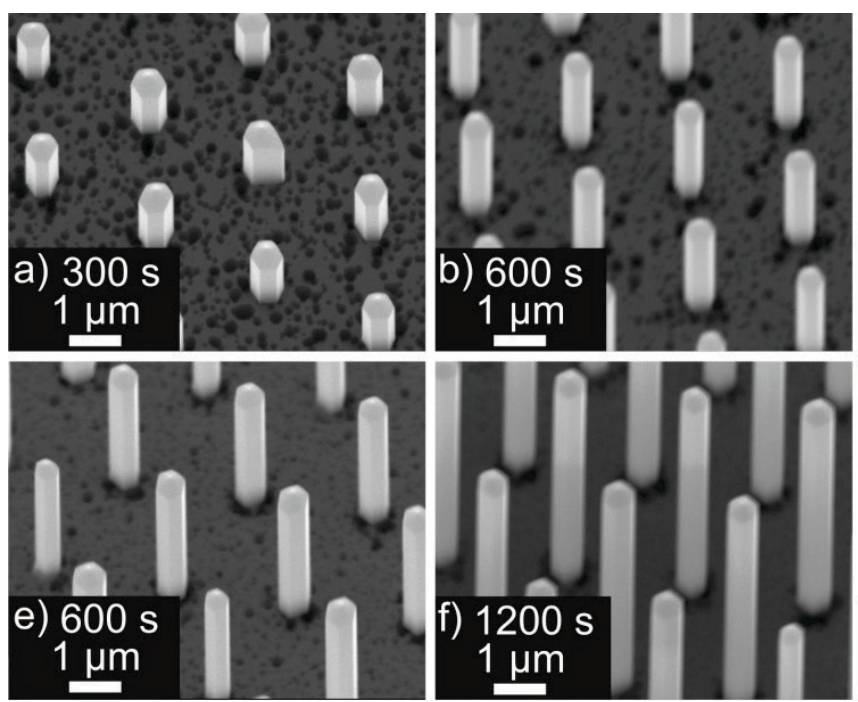
Figure $1 \mathrm{SE}$ images (EHT of $20 \mathrm{kV}$ ) under $30^{\circ}$ tilt of GaN columns grown without silane in the upper row for $300 \mathrm{~s}(\mathrm{a}), 600 \mathrm{~s}(\mathrm{~b})$,
$1200 \mathrm{~s}(\mathrm{c})$ and $1800 \mathrm{~s}(\mathrm{~d})$ and for GaN columns grown with a silane flow of $110 \mathrm{nmol} / \mathrm{min}$ in the lower row for $600 \mathrm{~s}(\mathrm{e}), 1200 \mathrm{~s}(\mathrm{f})$, $2400 \mathrm{~s}(\mathrm{~g})$ and $3600 \mathrm{~s}(\mathrm{~h})$. constant up to a height of $3.4 \mu \mathrm{m}$ of the columns, as can be seen in Fig. 2 (intersection of the two linear fits). Beyond this, the vertical growth rate saturates at the much lower value of $0.3 \mu \mathrm{m} / \mathrm{h}$. Applying silane during the growth leads - besides an enhanced n-type doping of the columns - to a drastically increased vertical growth rate, which has been reported before [11],[12],[13],[10]. In this case, a silane flow of $110 \mathrm{nmol} / \mathrm{min}$ led to a growth rate of approximately $23 \mu \mathrm{m} / \mathrm{h}$, but only during a first phase of microrod growth, where the height of the microrod was below 8.6 $\mu \mathrm{m}$ in this particular experiment. Interestingly, the growth rate beyond that was not close to zero like in the case with no silane, but was still as high as $8.5 \mu \mathrm{m} / \mathrm{h}$. This implies that the saturation of the growth rate could at least be partly overcome by an appropriate silane flow. Passing 330 $\mathrm{nmol} / \mathrm{min}$ silane into the reactor increased the vertical growth rate even to values of around $41 \mu \mathrm{m} / \mathrm{h}$. Up to a growth time of $2700 \mathrm{~s}$, corresponding to a height of $28 \mu \mathrm{m}$, no clear saturation of the growth rate was observed. The diameter of the rods was almost constant for the investigated growth times, if a silane flow of up to 110 $\mathrm{nmol} / \mathrm{min}$ was used. Nevertheless, for the highest applied silane flow of $330 \mathrm{nmol} / \mathrm{min}$, the diameter increased towards the tip of the columns (by $24 \mathrm{~nm}$ per $1 \mu \mathrm{m}$ height) and at the same time the height homogeneity of the columns decreased. Less selectivity [11] or increased height inhomogeneity [10] with enhanced $\mathrm{SiH}_{4}$ flow were also reported before. In our case, approximately every fourth hole $(28 \%)$ of the mask was not filled by a GaN column at this high silane flow. These missing rods have not been taking into account for the statistics in Fig. 2.

In principle there are three contributions to the vertical growth rate during SAG [14],[15]: the net flux (i.e. the difference between adsorbed and desorbed species on the surface) of growth species directly impinging from the gas
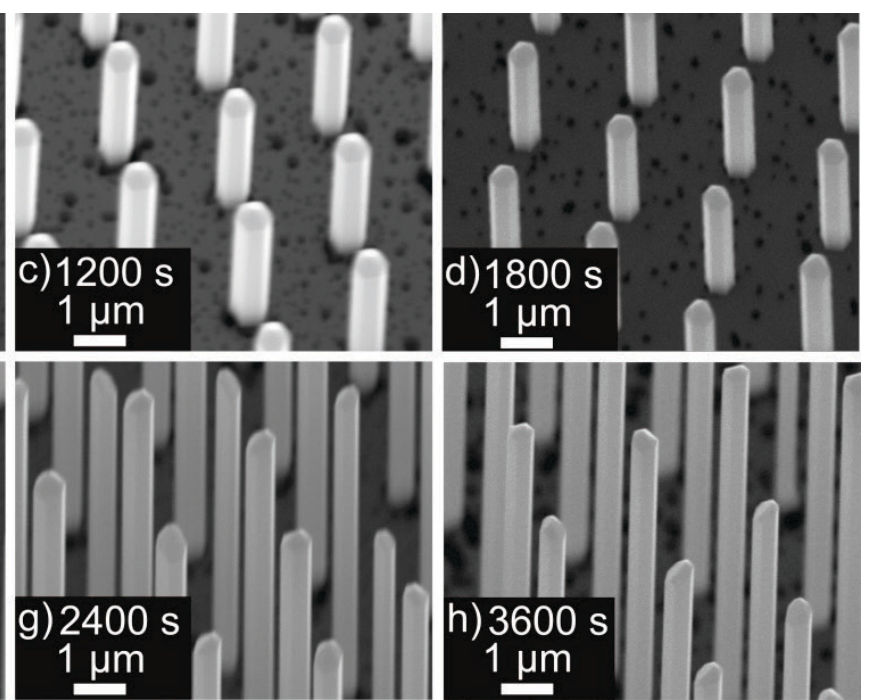


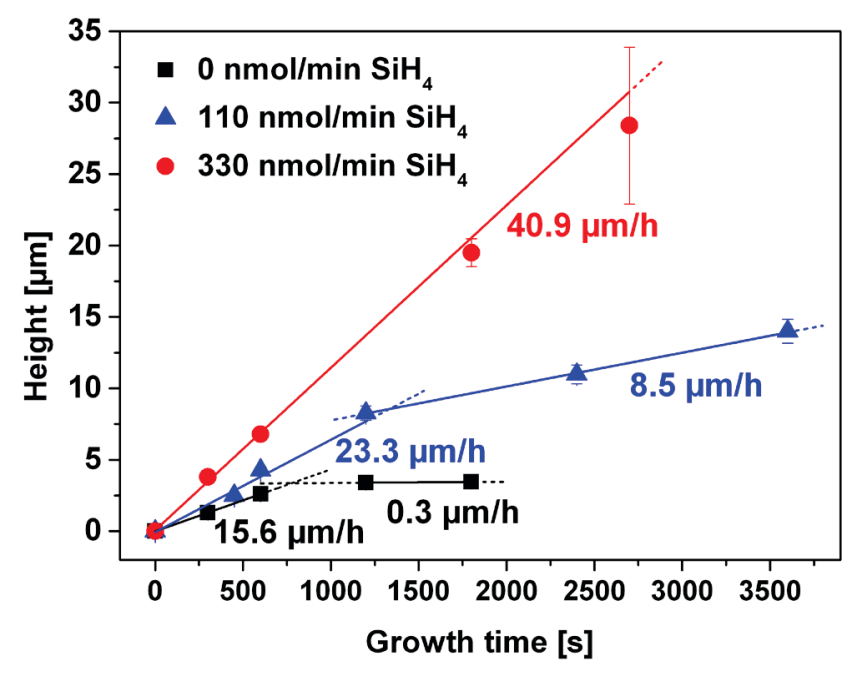

Figure 2 Dependence of height of the $\mathrm{n}-\mathrm{GaN}$ columns on growth time, the samples were grown with no silane (black squares), with a silane flow of $110 \mathrm{nmol} / \mathrm{min}$ (blue triangles) and with 330 $\mathrm{nmol} / \mathrm{min}$ (red circles), respectively.

phase on the top surface of the growing rods $\left(\mathrm{J}_{\text {top }}\right)$, the net flux impinging on the sidewalls $\left(\mathrm{J}_{\mathrm{sw}}\right)$ and diffusing along the sidewall to the top of the column, and the net flux of species impinging on the $\mathrm{SiO}_{\mathrm{x}}$ mask and diffusing from the $\mathrm{SiO}_{\mathrm{x}}$ mask to the rods $\left(\mathrm{J}_{\text {sub }}\right)$ [15]. The contribution of $\mathrm{J}_{\text {sub }}$ and $\mathrm{J}_{\mathrm{sw}}$ is based on the effective collection range, which is defined as $r$ here. If species impinge in a distance smaller than $r$ on the substrate/on the sidewalls they can contribute to the vertical growth rate. The effective collection range is probably mainly corresponding to the diffusion length of the species on the surface but it also takes into account the diffusion length in the gas phase (species could desorb from the surface, diffuse via the gas phase and be incorporated at the top facet) and also the sink efficiency, i.e. the incorporation efficiency.

Using this model of vertical growth and having in mind the saturation of growth rate beyond certain critical column heights, the differences in the growth rates may be assumed to originate from the limitation of the effective collection range of species on the column sidewalls, called $\mathrm{r}_{\mathrm{sw}}$, as discussed in the following.

$\mathrm{J}_{\text {top }}$ should stay constant during the growth time since the diameter of the columns was not increasing distinctly, at least for a silane flow of $110 \mathrm{nmol} / \mathrm{min}$ or less (see Fig. 1 , statistics are not shown here). The diffusion length of the precursors on the $\mathrm{SiO}_{\mathrm{x}}$ surface was determined for $\mathrm{N}$ polar column growth to be nearly independent of the silane flow and in the range of $1.3-1.6 \mu \mathrm{m}$ [15]. As the growth parameters do not vary substantially between Ga-polar and $\mathrm{N}$-polar growth regimes, it is assumed here, that the effective collection range on the growth mask $\left(\mathrm{r}_{\text {sub }}\right)$ is comparable for both cases. Since the distance between the columns in the investigated pattern was $1.6 \mu \mathrm{m}$, all the material that was contributing to the flux $\mathrm{J}_{\text {sub }}$ could diffuse to one of the adjacent rods. As the pitch did not vary over the evaluated samples, this flow of material was constant, and therefore it is unlikely that variations in $r_{\text {sub }}$ are the reason for the observed silane influence. The silane-dependent parameter is the collection range along the sidewalls, $r_{\text {sw }}$. If the rod height is larger than $r_{s w}$, the species coming from the substrate and coming from the lower part of the sidewalls will not contribute to the vertical growth rate any more. The vertical growth rate will decrease at this point due to the vanishing contribution of $\mathrm{J}_{\text {sub }}$ and/or the limited contribution of $\mathrm{J}_{\mathrm{sw}}$. As a consequence, using the model described, we can conclude that $r_{\mathrm{sw}}$ is increasing with the applied silane flow (see Fig. 2).

In order to determine $r_{s w}$ quantitatively, the height versus time plot could be fitted with the growth model of Ruth and Hirth [14], which is based on the three contributions $\mathrm{J}_{\mathrm{top}}, \mathrm{J}_{\mathrm{sw}}$ and $\mathrm{J}_{\mathrm{sub}}$. The insufficient number of data points does not allow the calculation of accurate values in this case. Nevertheless, it can be assessed that the fit would have an exponential behavior at the beginning (for 0 and $110 \mathrm{nmol} / \mathrm{min} \mathrm{SiH}_{4}$ ), followed by a linear behavior. As discussed by Dayeh et al. [16] for InAs nanowires, the substrate-wire exchange rate can be described by a factor $\beta$ with $\beta \propto \lambda_{\text {sub }} / \lambda_{\text {sw }}[14],[16]$ with $\lambda_{\text {sub }}$ and $\lambda_{\text {sw }}$ being the diffusion lengths on the substrate and on the wire sidewalls, respectively. Low $\beta$ factors represent a low substrate-wire exchange rate of species. The factor can vary considerably by the changing the growth conditions, e.g. the V/III ratio [16]. For GaN rods grown on $\mathrm{SiN}_{\mathrm{x}}$ patternd sapphire, a $\beta$ of 0.1 was found [17] whereas in our experiments $\beta$ should be much larger than 1 (due to the exponential increase at the beginning), meaning a relatively high growth rate contribution of the species impinging on the mask surface. This difference in the $\beta$ values could be explained by different growth conditions in different reactors but also by the different passivation layers. Possibly, the substrate-wire exchange rate is higher on $\mathrm{SiO}_{\mathrm{x}}$ than on $\mathrm{SiN}_{\mathrm{x}}$ surfaces.

For our experiments, a rough estimation of $r_{s w}$ was done by the intersection of the two linear fits for the fast and the slow vertical growth rate regime: According to that, $\mathrm{r}_{\mathrm{sw}}$ is $3.4 \mu \mathrm{m}$ without silane whereas it increased to $8.6 \mu \mathrm{m}$ for $110 \mathrm{nmol} / \mathrm{min}$ of $\mathrm{SiH}_{4}$. This value corresponds well to the average sidewall diffusion length of $7.5 \mu \mathrm{m}$ reported by Eymery [17] for self-assembled GaN columns grown with $\mathrm{SiH}_{4}$ on a $\mathrm{SiN}_{\mathrm{x}}$ passivation mask. For the highest silane flow of $330 \mathrm{nmol} / \mathrm{min}, \mathrm{r}_{\mathrm{sw}}$ seems to be $28 \mu \mathrm{m}$ or even higher (no clear kink observed up to a growth time of 2700 s). These findings show that an increased collection range due to higher silane flow might be the reason for an increased vertical growth rate and a delayed saturation of the growth rate over time.

In the case of very high silane flow (330 nmol/min), the growth rate was distinctly increased (about a factor of 2.6 compared to the growth rate without silane). This could be a possible explanation of the afore-mentioned structure with an increasing diameter towards the top of the rods. Since a large amount of material is diffusing up to the top facet, the reactions on the top of the rods could be slower 
than the species injection rate and not all of the material can be incorporated at the top facets. This could enforce lateral growth also on the sidewalls, with the consequence of a monotonously increasing diameter as described before.

The three different $\mathrm{SiH}_{4}$ flows not only led to different effective collection ranges, but also to different doping concentrations. Since direct Hall measurements are not readily possible in a $3 \mathrm{D}$ geometry, the doping concentrations have been estimated from the line width of the near band-edge emission peak of the GaN rods measured by room temperature photoluminescence as suggested by Yoshikawa et al. [18]. From that analysis, it was derived that $\mathrm{GaN}$ rods grown with a silane flow of $110 \mathrm{nmol} / \mathrm{min}$ and $330 \mathrm{nmol} / \mathrm{min}$ have a carrier concentration of about $6 \mathrm{x}$ $10^{19} \mathrm{~cm}^{-3}$ and $1.1 \times 10^{20} \mathrm{~cm}^{-3}$, respectively. More details about this will be published elsewhere.

\section{Detection of Si-rich surface layer}

In order to investigate the reason of the increased effective collection range with higher $\mathrm{SiH}_{4}$ flow, the surface of the $\mathrm{GaN}$ rods was investigated by transmission electron microscopy (TEM), energy dispersive x-ray spectroscopy (EDX) and Auger spectroscopy. The transition from the GaN core to an outer shell (in this case MQW) corresponds to the surface of the GaN rods after the 3D growth. Hence, a sample with an initial silane flow of $165 \mathrm{nmol} / \mathrm{min}$ during $\mathrm{GaN}$ core growth $(165 \mathrm{nmol} / \mathrm{min}$ for the first $650 \mathrm{~s}$ followed by $950 \mathrm{~s}$ without $\mathrm{SiH}_{4}$ ) and a 3 -fold $\mathrm{InGaN} / \mathrm{GaN}$ MQW shell embedded by an undoped GaN shell was investigated by TEM as well as EDX. In total the shell had a thickness of $80 \mathrm{~nm}$. The sample was prepared by focused ion beam etching (FIB). A scanning transmission electron microscope (STEM) image is shown in Fig. 3 a. The three QWs and one additional interface located more inside of the column (to the right) are clearly visible. By the EDX mapping $\mathrm{Ga}$ and $\mathrm{N}$ could be detected in the whole GaN column (Fig. 3 e-f), as expected. Au and Pt from the sample preparation exist outside of the column (on the left side in Fig. 3 b-c). The mapping of Si (Fig. 3 d) shows some $\mathrm{Si}$ signal in the areas surrounding the rods, which is a measurements artifact due to the increased background scattering in the $\mathrm{Au} / \mathrm{Pt}$. Besides of this, an increased Si concentration could also be detected in the afore-mentioned interface inside of the column. This interface corresponds to the transition from the vertically grown GaN core to the laterally grown shell which starts with an undoped GaN spacer layer. This is an indication that the pure GaN columns have a Si-rich layer on their sidewall facets, which might increase the effective diffusion lengths of species along the sidewalls. A Si-rich layer at the sidewalls (in this case $\mathrm{SiN}$ ) was already reported by Tessarek at el. [13] for selforganized grown GaN rods. A patent by Eymery et al. [19] describes the in-situ fabrication of a thin $\mathrm{SiN}_{\mathrm{x}}$ layer on $\mathrm{GaN}$ wire sidewalls and the capability of using this layer as a passivation [20] or insulation layer for device fabrication.

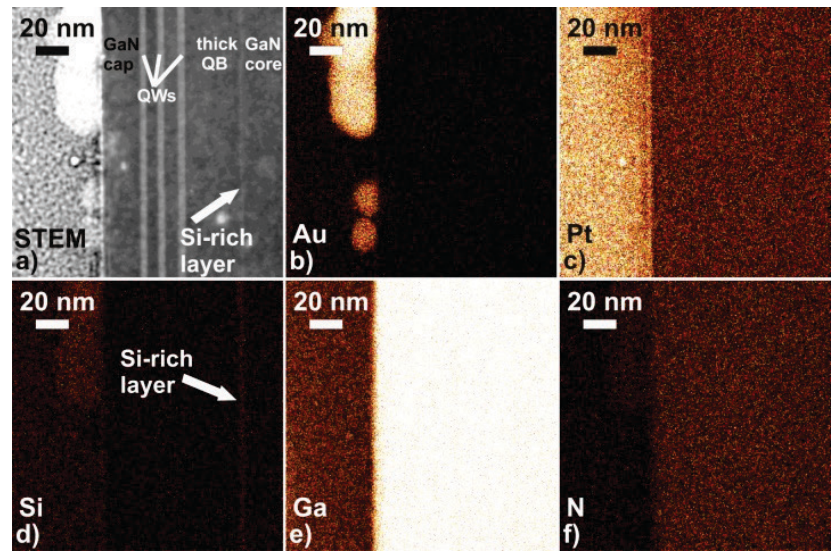

Figure 3 STEM (a) and corresponding EDX mappings for the elements $\mathrm{Au}(\mathrm{b}), \mathrm{Pt}$ (c), Si (d), Ga (e) and N (f) on one sidewall of a $3 \mathrm{D}-\mathrm{GaN}$ core-shell structure.

Judging from the EDX mapping, the Si-rich layer has a thickness of less than $2 \mathrm{~nm}$ in the structure shown here. The fact that this layer was detected by EDX indicates very high concentration levels of silicon.

In order to quantify the amount of silicon at the side facets of the microrods, the Si-rich layer was also investigated by Auger electron spectroscopy. GaN cores were grown with a silane flow of $165 \mathrm{nmol} / \mathrm{min}$ and 330 $\mathrm{nmol} / \mathrm{min}$, respectively. To avoid Auger electron emission from the substrate induced by backscattered electrons and to rule out $\mathrm{Si}$ signals from the $\mathrm{SiO}_{\mathrm{x}}$ mask, some of the microrods have been detached from their substrate and were deposited on a gold-coated silicon wafer. Auger electron spectra of side facets of the rods reveal, besides the main components $\mathrm{Ga}$ and $\mathrm{N}$ and growth process-related $\mathrm{Si}$, signals due to $\mathrm{O}$ and $\mathrm{C}$ contaminations (Fig. 4 a). The $\mathrm{O}$ and $\mathrm{C}$ contaminations are at least partially due to the transport of the samples through the air. Auger spectra for a detached single rod grown with $330 \mathrm{nmol} / \mathrm{min}$ silane have been taken at five points along the rod axis (see scheme in Table 1). Using a standard procedure described by Seah [21] and sensitivity factors for the individual Auger transitions recommended by the instrument's manufacturer, nominal concentrations of the relevant elements were derived from the differentiated Auger spectra at the five points (Table 1).

It should be noted, however, that the evaluation of elemental concentrations from Auger spectra according to Seah [21] is strictly valid only for material systems with homogeneous chemical composition. We have demonstrated the principal applicability of this procedure for such systems by an Auger analysis of the clean and non-polar surfaces of GaN yielding almost equal atomic concentrations for $\mathrm{Ga}$ and $\mathrm{N}$ (data not shown). Taking into account that the detected $\mathrm{Si}$ is silane-induced (see below) and very likely forms a thin surface layer on our GaN microrods, and taking into account that the $\mathrm{C}$ and $\mathrm{O}$ signals are likely due to (post) growth process contamination, we expect that the chemical composition at the surface of the microrod is 
Table 1 Nominal concentrations [\%] of elements.

\begin{tabular}{|c|c|c|c|c|c|}
\hline $\begin{array}{l}\begin{array}{l}\text { Regi- } \\
\text { on* }\end{array} \\
\end{array}$ & $\begin{array}{c}\mathrm{Si}- \\
\text { KLL }\end{array}$ & $\mathrm{Ga}$ & $\mathrm{N}$ & $\mathrm{O}$ & C \\
\hline 1 & 6.4 & 37.6 & 23.9 & 5.3 & 26.8 \\
\hline 2 & 6.2 & 40.6 & 25.7 & 3.7 & 23.7 \\
\hline 3 & 8.6 & 36.2 & 22.9 & 4.7 & 27.7 \\
\hline 4 & 7.2 & 28.5 & 12.5 & 6.6 & 44.9 \\
\hline 5 & 7.6 & 24.9 & 9.3 & 5.8 & 52.4 \\
\hline
\end{tabular}

Calculated concentrations (see text):

$\begin{array}{llllll}1 & 5.7 & 37.0 & 28.2 & 5.0 & 24.0 \\ 5 & 8.9 & 22.9 & 11.4 & 6.0 & 50.8\end{array}$

* Nominal concentrations [\%] of elements measured by Auger electron spectroscopy at spots 1 to 5 on a side facet of an n-GaN microrod. Calculated nominal concentrations for thicknessgraded layers of $\mathrm{Si}$ and $\mathrm{C} / \mathrm{O}$ on-top of the $\mathrm{GaN}$ facet are indicated for spots 1 and 5 .

not homogeneous but largely layered. The graphite-like lineshape of the $\mathrm{C}$ Auger signal adds further support for a dense carbon layer. $\mathrm{O}$ and a corresponding minor $\mathrm{C}$ contribution are tentatively assigned to surface $\mathrm{CO}$ contamination on-top of this $\mathrm{C}$ layer. For a more refined analysis of surface concentrations, attenuation of Auger signals from deeper regions of the surface has to be taken into account. Therefore, we have calculated the Auger signals and nominal concentrations assuming the following sequence of layers for our sample: $\mathrm{GaN}$ bulk (representing the microrod), a thin Si film and a contamination layer on-top of the $\mathrm{Si}$ film which mainly consists of $\mathrm{C}$ and a small amount of $\mathrm{O}$. The attenuation of the Auger electrons from layers beneath the surface is included in our calculations by exponential attenuation with the inelastic mean free path as decay constant [22],[23]. The different strengths of the Auger transitions of the elements involved are taken into account by using the same sensitivity factors that we use for the Auger data processing. The surface layer contains a submonolayer amount of oxygen which is not included in the calculation of the attenuation of the subsurface layers as the influence of an exchange of $\mathrm{C}$ atoms by $\mathrm{O}$ atoms in the model is negligible. By comparison of the calculated results for the layered stack with the concentrations derived from the measurements we are able to provide estimates for the thicknesses of the individual layers. We find good agreement between measured and calculated nominal concentrations (see Table 1) for the following thicknesses:

a) For spot 1 in the sketch of Table 1 (top of the microrod): GaN bulk +1 atomic monolayer (ML) $\mathrm{Si}$ +1 ML carbon.

b) For spot 5 in the sketch of Table 1 (bottom of the microrod): $\mathrm{GaN}$ bulk +2 atomic monolayers (ML) $\mathrm{Si}+3 \mathrm{ML}$ carbon.

Calculated and measured nominal concentrations agree within a margin of $10 \%$. Only the $\mathrm{N}$ concentration and the $\mathrm{Si}$ concentration at the bottom appear to be overestimated in the calculations. The calculated $\mathrm{N}$ concentration is $20 \%$ higher than the measured one. This deviation can be explained by the Ga-rich growth conditions for the microrods which may lead to a Ga adlayer on the GaN side facets [24]. The overestimated $\mathrm{Si}$ concentration at the bottom is probably caused by a less dense Si. Overall, we find a Si adlayer with decreasing thickness from the bottom of the rod to the top. This Si gradient along the axis of the rods is consistent with the findings by Tessarek et al. [13]. The impact of the gradient, i.e. a low deposition rate at the bottom of the GaN wires, was also shown by Koester et al. [7].
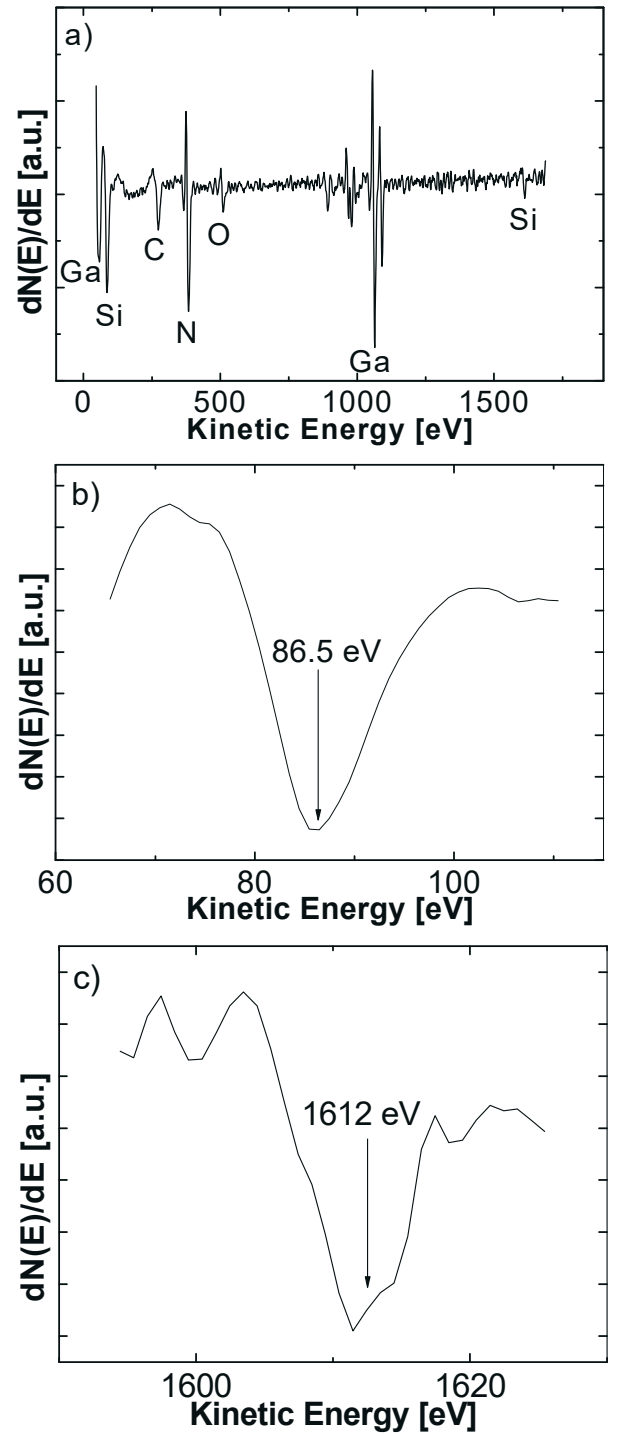

Figure 4 Differential Auger electron spectra measured at the center of the sidewall surface of an $\mathrm{n}-\mathrm{GaN}$ core grown with 165 $\mathrm{nmol} / \mathrm{min}$ of $\mathrm{SiH}_{4}$ : (a) overall spectrum, (b) LVV Auger transition of Si (c) KLL Auger transition of Si. 
Auger spectra of a nominally undoped rod which was not subjected to silane exposure did not reveal any Si signals. Therefore, the Si-rich surface layer on the microrods is definitively related to the introduction of silane during the MOVPE growth process and not to desorption of silicon from the $\mathrm{SiO}_{x}$ mask material. The $\mathrm{Si}$ gradient can be explained by a longer effective exposure of the bottom part of the rod to the silane-containing process gas in the reactor favoring a stronger accumulation of $\mathrm{Si}$ at the bottom part of the side facets.

The carbon contamination layer is also thicker at the bottom and presumably caused by organic residuals of the growth process on the Si layer, since a concentration gradient along the rod length is unlikely to be caused by an atmospheric contamination. The total thickness of the adlayers $(\mathrm{Si}+\mathrm{C} / \mathrm{O})$ as suggested by the above model calculations is between $0.6 \mathrm{~nm}$ (top) and $1.5 \mathrm{~nm}$ (bottom). This range of thicknesses was confirmed by sputter cleaning of the samples using an $\mathrm{Ar}^{+}$ion beam: after removal of a layer with a thickness of about $1-2 \mathrm{~nm}$ both contamination signals and $\mathrm{Si}$ signals vanished.

Further information concerning the Si-rich layer can be deduced from the peak positions of the Si-LVV and the SiKLL Auger transition. The tabulated transitions for elemental $\mathrm{Si}$ are $92 \mathrm{eV}$ and $1619 \mathrm{eV}$ respectively, for $\mathrm{Si}$ in $\mathrm{SiO}_{2}$ one finds $76 \mathrm{eV}$ and $1606 \mathrm{eV}$, respectively [25]. Thus, with positions between $86.5 \mathrm{eV}$ to $91 \mathrm{eV}$ and $1612 \mathrm{eV}$ to $1614 \mathrm{eV}$ measured in this report (Fig. 4 b,c) the $\mathrm{Si}$ is found to be far from higher oxidation levels. The experimental results fit better to energies reported for $\mathrm{SiN}_{\mathrm{x}}$ [26] thin films and to Si-C bonds [27] in accordance to our layer model.

The Si-rich layer could be removed by wet chemical etching with hot phosphoric acid $\left(85 \%, 150^{\circ} \mathrm{C}, 10 \mathrm{~s}\right)$. This has been confirmed by surface photovoltage measurements [28] as well as by Auger measurements on the sidewalls after the etching step, which showed a drastically reduced Si signal.

4 Suggested growth mechanism The above results suggest the following SAG mechanism of the GaN columns: Species from the gas phase impinge on the substrate and diffuse to the open windows in the $\mathrm{SiO}_{\mathrm{x}}$ mask $\left(\mathrm{J}_{\mathrm{sub}}\right)$. GaN nuclei form and finally grow towards pyramids (this is assumed on the basis of TEM measurements which are not shown here). During this filling of the mask holes, a high V/III ratio is used that is comparable to layer growth conditions. Therefore, the sidewalls of the pyramids are passivated by the reactive hydrogen, coming from the high amount of $\mathrm{NH}_{3}$ in the growth atmosphere [29]. Thereafter, the growth conditions are changed to a three dimensional growth mode with a low V/III ratio to remove the hydrogen passivation of the pyramid sidewalls by the increased concentration of Ga-containing species [29]. This process thus promotes the growth on the semi-polar pyramid facets. Since the lateral growth is constrained due to the $\mathrm{SiO}_{x}$ mask and since the growth rate in m direction is very small, non-polar sidewalls are formed at the rim of the mask holes. Consequently, most of the Ga species might either diffuse upwards along the sidewalls to be incorporated in one of the top facets or they desorb from the sidewalls. Just a negligible amount of $\mathrm{Ga}$ is built into the sidewall facets so that the average diameter of the columns remains almost constant over the growth time (for silane flows up to 110 $\mathrm{nmol} / \mathrm{min}$ ) which is in accordance with the experimental findings. With the added silane in the growth atmosphere, there is also a minor reaction of $\mathrm{Si}$ with $\mathrm{N}$ at the non-polar sidewalls. Once this very thin Si-rich layer has emerged, the surface atom structure is changed. This could be a probable explanation for the enhanced collection range of the species. Additionally, the Si-rich layer can prevent the $\mathrm{Ga}$ incorporation on the sidewall, like an in-situ $\mathrm{SiN}_{\mathrm{x}}$ mask which is used for dislocation reduction of GaN [30]. Although the growth of $\mathrm{GaN}$ is prevented under these growth conditions, the $\mathrm{Si}$ accumulates on the side facets and is incorporated forming a Si-rich layer with a thickness increasing gradually with growth time. This explains the observed gradient of $\mathrm{Si}$ in the Auger results.

For very high rods with a fast growth rate (using 330 $\mathrm{nmol} / \mathrm{min}$ silane) the Si-rich layer at the top is not as pronounced as compared to the bottom which might be due to less thickness or lower Si concentration. Therefore some Ga incorporations on the sidewalls could occur on the upper part of the rods despite the Si-rich layer which could be another reason for the increasing diameter of these rods along the growth axis.

5 Conclusion We investigated the influence of silane on the MOVPE growth rate of selectively grown GaN columns. Silane also leads to a modified surface of the GaN structures. The Si-rich surface layer was detected by EDX and Auger spectroscopy on the sidewalls especially at the lower part of the rods. Presumably, this additional enclosure affects the reactions at the sidewalls as well as the diffusion of species and it increases the effective collection range which in turn defers the saturation of growth rate vs. time and allows more material to reach the top of the rods. There the local concentration of growth relevant species is enhanced and the vertical growth rate is increased drastically. The Si-rich passivation layer has substantial impact on the further growth of shell layers. It can be either practicable (as a passivation or insulation layer) or unfavorable for device fabrication since no homogenous, high quality MQW can be grown along the whole sidewall height.

Acknowledgements The authors acknowledge the financial support of DFG research unit 1616 (Dynamics and Interactions of Semiconductor Nanowires for Optoelectronics), the Niedersächsische Technische Hochschule (NTH) and the EU project "GECCO" (Project No. 280694). We thank Prof. M. Schilling for allowing us to use his FE-SEM. 


\section{References}

[1] A. Waag, X. Wang, S. Fündling, J. Ledig, M. Erenburg, R. Neumann, M. Al Suleiman, S. Merzsch, J. Wei, S. Li, H.H. Wehmann, W. Bergbauer, M. Straßburg, A. Trampert, U. Jahn, H. Riechert, Phys. Status Solidi Curr. Top. Solid State Phys. 8 (2011) 2296.

[2] Y.J. Hong, C.-H. Lee, A. Yoon, M. Kim, H.-K. Seong, H.J. Chung, C. Sone, Y.J. Park, G.-C. Yi, Adv. Mater. 23 (2011) 3284.

[3] Y.B. Tang, Z.H. Chen, H.S. Song, C.S. Lee, H.T. Cong, H.M. Cheng, W.J. Zhang, I. Bello, S.T. Lee, Nano Lett. 8 (2008) 4191.

[4] L. Caccamo, J. Hartmann, C. Fàbrega, S. Estradé, G. Lilienkamp, J.D. Prades, M.W.G. Hoffmann, J. Ledig, A. Wagner, X. Wang, L. Lopez-Conesa, F. Peiró, J.M. Rebled, H.-H. Wehmann, W. Daum, H. Shen, A. Waag, ACS Appl. Mater. Interfaces 6 (2014) 2235.

[5] Y. Huang, X. Duan, Y. Cui, C.M. Lieber, Nano Lett. 2 (2002) 101

[6] H. Wu, H.Y. Cha, M. Chandrashekhar, M.G. Spencer, G. Koley, J. Electron. Mater. 35 (2006) 670.

[7] R. Koester, J.S. Hwang, D. Salomon, X. Chen, C. Bougerol, J.P. Barnes, D.L.S. Dang, L. Rigutti, A. De Luna Bugallo, G. Jacopin, M. Tchernycheva, C. Durand, J. Eymery, Nano Lett. 11 (2011) 4839.

[8] K.-C. Kim, M.C. Schmidt, H. Sato, F. Wu, N. Fellows, Z. Jia, M. Saito, S. Nakamura, S.P. DenBaars, J.S. Speck, K. Fujito, Appl. Phys. Lett. 91 (2007) 181120.

[9] X. Li, X. Ni, J. Lee, M. Wu, Ü. Özgür, H. Morkoç, T. Paskova, G. Mulholland, K.R. Evans, Appl. Phys. Lett. 95 (2009) 121107.

[10] X. Wang, S. Li, M.S. Mohajerani, J. Ledig, H.H. Wehmann, M. Mandl, M. Strassburg, U. Steegmüller, U. Jahn, J. Lähnemann, H. Riechert, I. Griffiths, D. Cherns, A. Waag, Cryst. Growth Des. 13 (2013) 3475.

[11] S. Haffouz, B. Beaumont, P. Gibart, MRS Internet J. Nitride Semicond. Res. 3 (1998).

[12] R. Koester, J.S. Hwang, C. Durand, D.L.S. Dang, J. Eymery, Nanotechnology 21 (2010) 015602.

[13] C. Tessarek, M. Heilmann, E. Butzen, a. Haab, H. Hardtdegen, C. Dieker, E. Spiecker, S. Christiansen, Cryst. Growth Des. 14 (2014) 1486.

[14] V. Ruth, J.P. Hirth, J. Chem. Phys. 41 (1964) 3139.

[15] X. Wang, J. Hartmann, M. Mandl, M. Sadat Mohajerani, H.-H. Wehmann, M. Strassburg, A. Waag, J. Appl. Phys. 115 (2014) 163104.

[16] S.A. Dayeh, E.T. Yu, D. Wang, Nano Lett. 9 (2009) 1967.

[17] J. Eymery, X. Chen, C. Durand, M. Kolb, G. Richter, Comptes Rendus Phys. 14 (2013) 221.

[18] M. Yoshikawa, M. Kunzer, J. Wagner, H. Obloh, P. Schlotter, R. Schmidt, N. Herres, U. Kaufmann, J. Appl. Phys. 86 (1999) 4400.

[19] J. Eymery, D. Salomon, X. Chen, C. Durand, Method of Selective Growth without Catalyst on a Semiconductor Struture, Patent US 2014/0080290 A1, 2014.

[20] M. Tchernycheva, A. Messanvi, A. de Luna Bugallo, G. Jacopin, P. Lavenus, L. Rigutti, H. Zhang, Y. Halioua, F.H. Julien, J. Eymery, C. Durand, Nano Lett. 14 (2014) 3515.
[21] P. Seah, in:, Surf. Anal. by Auger X-Ray Photoelectron Spectrosc. by David Briggs, John T. Grant, IM Publications LLP, 2003.

[22] NIST Standard Reference Database 71, http://www.nist.gov/srd/nist71.cfm.

[23] S. Tanuma, C.J. Powell, D.R. Penn, Surf. Interface Anal. 37 (2005) 1.

[24] C.E. Dreyer, A. Janotti, C.G. Van de Walle, Phys. Rev. B 89 (2014) 081305.

[25] L.E. Davis, Handbook of Auger Electron Spectroscopy: A Reference Book of Standard Data for Identification and Interpretation of Auger Electron Spectroscopy Data, Physical Electronics Industries, Minnesota, 1976.

[26] J.A. Taylor, Appl. Surf. Sci. 7 (1981) 168.

[27] L.I. Johansson, C. Virojanadara, T. Eickhoff, W. Drube, Surf. Sci. 529 (2003) 515.

[28] M.A. Deeb, J. Wei, J. Hartmann, H.-H. Wehmann, A. Waag, Phys. Status Solidi 212 (2015) 732.

[29] S. Li, A. Waag, J. Appl. Phys. 111 (2012) 071101.

[30] D.-K. Kim, J. Korean Phys. Soc. 51 (2007) 1718. 